\title{
Order-to-chaos transition in the model of quantum pendulum subjected to noisy perturbation
}

\author{
D. V. Makarov, L. E. Kon'kov \\ Laboratory of Nonlinear Dynamical Systems, \\ Pacific Oceanological Institute of the Russian Academy of Sciences, \\ 43 Baltiiskaya st., 690041 Vladivostok, Russia, URL: http://dynalab.poi.dvo.ru
}

\begin{abstract}
.
Motion of randomly-driven quantum nonlinear pendulum is considered. Utilizing one-step Poincaré map, we demonstrate that classical phase space corresponding to a single realization of the random perturbation can involve domains of finite-time stability. Statistical analysis of the finite-time evolution operator (FTEO) is carried out in order to study influence of finite-time stability on quantum dynamics. It is shown that domains of finite-time stability give rise to ordered patterns in distributions of FTEO eigenfunctions. Transition to global chaos is accompanied by smearing of these patterns; however, some of their traces survive on relatively long timescales.
\end{abstract}

Keywords: finite-time stability, random perturbation, quantum chaos, one-step Poincaré map, finite-time evolution operator

\section{Introduction}

It is well-known that deterministic classical systems with few degrees of freedom can exhibit complicated chaotic behaviour that is similar to behaviour of systems with intrinsic random fluctuations. However, the methods used for studying deterministic chaotic systems and systems under stochastic driving differ substantially. Representing the external perturbation as some random process, we add uncertainty into the equations of motion. Each realization of the perturbation creates an unique trajectory of the system, therefore, efficient description of the system's response implies the usage of statistical analysis.

Nevertheless, single realizations of the random perturbation can be considered as deterministic functions. As long as the temporal Fourier spectrum of the perturbation is broad, there is no way for survival of impenetrable stable domains in phase space [1,2]. However, statistical analysis of the finite-time Lyapunov exponents in randomly-driven systems shows that remarkable fraction of phase space area maintains stability on timescales which exceed significantly the so-called Lyapunov time

$$
t_{\mathrm{L}}=\frac{1}{\lambda_{L}}
$$


where $\lambda_{L}$ is the global Lyapunov exponent calculated with $t \rightarrow \infty$ [3,4]. Such trajectories form bundles like branched flows in quantum point contacts [5] 8], or coherent ray clusters in ocean acoustics [9]. One shouldn't confuse this kind of coherent phenomena with the domains of particle clusterization in random fields [10]. The main difference is the location of initial conditions for particles. In the case of the coherent clusters, the initial conditions belong to continuous manifolds in phase space. Location of that manifolds depends on particular realization of the perturbation. Such manifolds can be found out in various ways. For example, one can calculate the map of finite-time Lyapunov exponents in phase space [11,12], or compute eigenfunctions of the transfer operator [13]. It turns out that system's behaviour under a single realization can significantly differ from the picture obtained via statistical averaging. There can occur phenomena which are typical for deterministic systems, for example, intermittency and capturing into dynamical traps [14-16]. Thus, one needs some general approach which can be used for both deterministic and noisy cases.

The problem of interrelation between deterministic and statistical approaches also arises in quantum systems whose classical counterparts exhibit chaotic behaviour. Periodic orbit theory [17, 18] provides classical interpretation of quantum spectra and, in addition, reveals some non-classical features, like scars of wavefunctions [19]. One can suggest that some peculiarities of deterministic quantum systems should manifest themselves in quantum systems involving classical noise. The issue of particular interest is how deterministic phenomena associated with periodic orbits are revealed under stochastic driving, when there is no periodic orbits in the strict sense.

In the present paper, we demonstrate approach that allows one to analyze quantum systems under weak random perturbation in the framework of deterministic theory. In that approach, it is implied that system's behaviour possesses some features which are common for all typical realizations of the perturbation. In this way, any realization is treated as a deterministic process with known spectral properties. On the classical level, our approach is based on the one-step Poincaré map originally introduced in [20,21]. It serves as a tool for finding out phase space patterns repeating themselves after some time interval. Some of these patterns have regular form and can be referred to as domains of finite-time stability. We use the quantum counterpart of the one-step Poincaré map, the so-called finite-time evolution operator, for exploring manifestations of finite-time stability in quantum motion. Mathematically equivalent approach was used for studying sound propagation in a randomly-inhomogeneous oceanic waveguide [22, 23. In the present paper, we use this approach for a purely quantum problem, namely for the quantum nonlinear pendulum subjected to broadband perturbation. Our main goal is to study how domains of finite-time stability influence quantum dynamics.

It is worth mentioning that the condition of invariance under translation over finite time interval is too restrictive, and there can be phase space patterns which don't satisfy it but correspond to non-chaotic dynamics. A striking example is the so-called branched flows in quantum point contacts [7,8]. Our approach is rather designed for oscillatory motion subjected to external noise. In particular, it can be implemented 
in the problems of noise-driven dissociation or ionization [24 26 , where regular phase space domains prevent transitions into unbounded states, therefore, efficient destruction of these domains is of great importance. The same problem arises in randomly-driven quantum ratchets with cold atoms [27].

The paper is organized as follows. The next section describes the model under consideration. In section 3 , we study classical motion of a randomly-driven pendulum by means of one-step Poincaré map. Signatures of classical finite-time stability in quantum dynamics are explored in section 4. In Conclusion, we summarize the results obtained.

\section{Model}

Consider the quantum Hamiltonian

$$
\begin{gathered}
\hat{H}=-\frac{\hbar^{2}}{2} \frac{\partial}{\partial x}+U(x)+\varepsilon V(x, t), \\
U(x)=-\cos x, \quad V(x, t)=f(t) \sin x-f(t+\Delta) \cos x
\end{gathered}
$$

where $\varepsilon \ll 1$, and $f(t)$ is so-called harmonic noise [28, 29]. The corresponding Schrödinger equation reads

$$
i \hbar \frac{\partial \Psi}{\partial t}=\hat{H} \Psi \text {. }
$$

In the present paper we simplify the analysis by considering only quantum states with zero quasimomentum. It corresponds to the periodic boundary conditions $\Psi(-\pi, t)=$ $\Psi(\pi, t)$. This simplification is quite reasonable if we deal with semiclassical regime when energy bands are flat, and tunneling between neighbouring potential wells is fairly weak. It demands the Planck constant to be small, therefore, we set $\hbar=0.1$. The model (2) was used in [27] in the context of quantum ratchet phenomena.

Harmonic noise is described by coupled stochastic differential equations

$$
\dot{f}=y, \quad \dot{y}=-\Gamma y-\omega_{0}^{2} f+\sqrt{2 \beta \Gamma} \xi(t),
$$

where $\Gamma$ is a positive constant, and $\xi(t)$ is Gaussian white noise. The terms $f(t)$ and $f(t+\Delta)$ in (2) correspond to identical realizations of harmonic noise and differ only by the temporal shift $\Delta$. The first two moments of harmonic noise are given by

$$
\langle f\rangle=0, \quad\left\langle f^{2}\right\rangle=\frac{\beta}{\omega_{0}^{2}} .
$$

We set $\beta=1$, that is, the perturbation strength is solely determined by the parameter $\varepsilon$. In the case of low values of $\Gamma$, the power spectrum of harmonic noise has the peak at the frequency

$$
\omega_{\mathrm{p}}=\sqrt{\omega_{0}^{2}-\frac{\Gamma^{2}}{2}} .
$$

Width of the peak is given by the formula

$$
\Delta \omega=\sqrt{\omega_{\mathrm{p}}+\Gamma \omega^{\prime}}-\sqrt{\omega_{\mathrm{p}}-\Gamma \omega^{\prime}}
$$


where $\omega^{\prime}=\sqrt{\omega_{0}^{2}-\Gamma^{2} / 4}$. As $\Gamma \rightarrow 0, f(t) \rightarrow \sin \left(\omega_{0} t+\phi_{0}\right)$, where $\phi_{0}$ is determined by initial conditions in (4). Setting $f(0)=1, y(0)=0$, and $\Delta=\pi /\left(2 \omega_{0}\right)$, one can easily find that $V(x, t)=\sin \left(x+\omega_{0} t\right)$ in the case of $\Gamma=0$. Hence, it turns out that, for $\Gamma>0, V(x, t)$ behaves like a plane wave whose amplitude and phase velocity fluctuate with time. The plane-wave acts as a dragging force for particles and leads to the onset of directed current, i. e. the ratchet effect. This kind of ratchets is known as travelling potential ratchets [30 33]. In the semiclassical regime, direction of the current coincides with the direction of the perturbation phase velocity, provided dynamical barriers preventing the transition of particles into infinite regime are destroyed. More intricate behaviour is observed in the deep quantum regime [34]. In the present work we use the following values of parameters: $\omega_{0}=1, \Gamma=0.1$ and $\varepsilon=0.05$.

\section{One-step Poincaré map}

Let's begin with the classical level and consider the classical counterpart of the Hamiltonian (2)

$$
H=\frac{p^{2}}{2}+U(x)+\varepsilon V(x, t) .
$$

Corresponding equations of motion read

$$
\frac{d x}{d t}=p, \quad \frac{d p}{d t}=-\frac{d U}{d x}-\varepsilon \frac{d V}{d x} .
$$

Let's consider some arbitrarily chosen realization of $V(x, t)$. Then we can treat $V(x, t)$ as a deterministic function and refer to (9) as the system of ordinary differential equations. As $V(x, t)$ is an oscillating function of time, the domains of finite-time stability may involve components which transform to themselves without mixing in course of evolution from $t=0$ to $t=\tau$. These components can be found out by means of the one-step Poincaré map [20, 21, 35]

$$
p_{i+1}=p\left(t=\tau ; p_{i}, x_{i}\right), \quad x_{i+1}=x\left(t=\tau ; p_{i}, x_{i}\right),
$$

where $p\left(t=\tau ; p_{i}, x_{i}\right)$ and $x\left(t=\tau ; p_{i}, x_{i}\right)$ are solutions of (9) with initial conditions $p(t=0)=p_{i}, x(t=0)=x_{i}$. One-step Poincaré map is equivalent to the usual Poincaré map with the Hamiltonian

$$
\begin{aligned}
& \bar{H}=\frac{p^{2}}{2}+U(x)+\varepsilon \tilde{V}(x, t), \\
& \tilde{V}(x, \bar{t}+n \tau)=V(x, \bar{t}), \quad 0 \leqslant \bar{t} \leqslant \tau,
\end{aligned}
$$

$n$ is an integer. Thus, we replace the original system by the equivalent time-periodic one. Validity of this replacement is provided by the time restriction to the interval $[0: \tau]$. An alternative approach for generalization of Poincaré map onto stochastic dynamical systems was offered in [36, 37].

Following the analogy with the usual Poincaré map, one can deduce the main

property of one-step Poincaré map: if a trajectory of (10) forms a closed continuous 

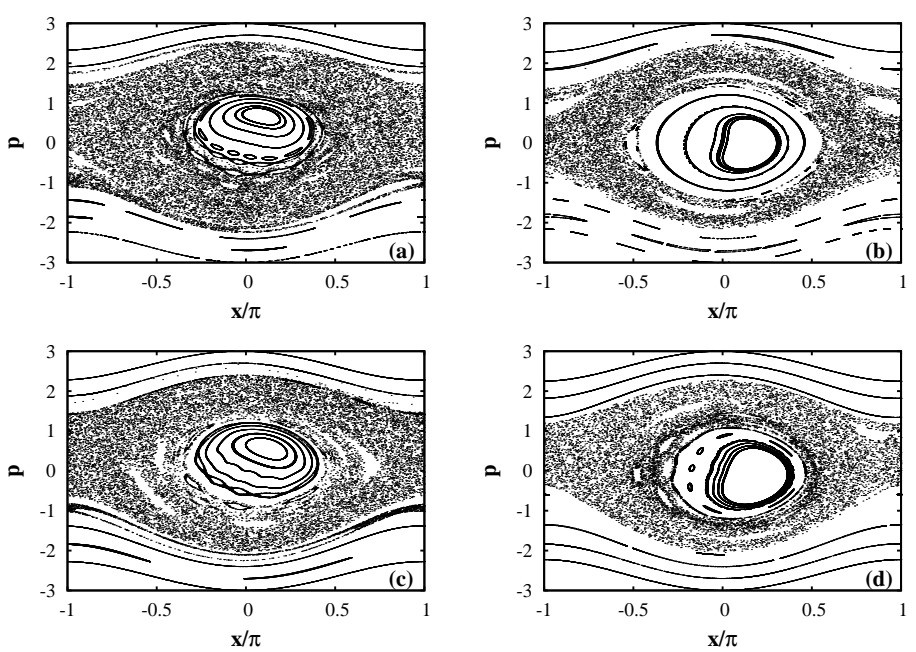

Figure 1. Phase space portraits constructed via one-step Poincaré map with $\tau=4 \pi$. Figures (a)-(d) correspond to different realizations of harmonic noise.
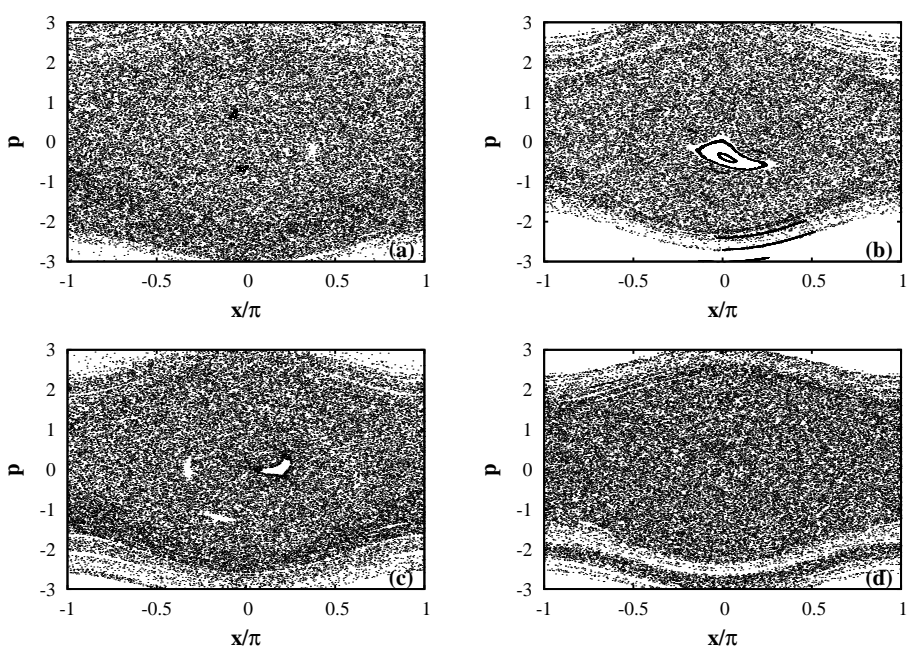

Figure 2. Phase space portraits constructed via one-step Poincaré map with $\tau=20 \pi$. Figures (a)-(d) correspond to different realizations of harmonic noise.

curve in phase space, any point belonging to it is an initial condition for a trajectory of (9) that remains stable at $t=\tau$. The inverse statement is not generally true, therefore, one-step Poincaré map yields a sufficient but not necessary criterion of stability. This means that one-step Poincaré map basically underestimates area of regular domains.

As we artificially reduce the problem to a time-periodic one, theory of time-periodic Hamiltonian systems can be invoked [20,21]. Phase space structure of one-step Poincaré map is determined by resonances

$$
m_{1} \tau=m_{2} T,
$$


where $T$ is the period of unperturbed motion. Period is function of the action variable defined as [14]

$$
I=\frac{1}{2 \pi} \oint p d x .
$$

Each resonance has certain width in the space of the action. Width can be calculated using the theory of nonlinear resonance. It can be shown that the distance between neighbouring dominant nonlinear resonances decreases with increasing $\tau$ as $\tau^{-1}$ [20]. This results in resonance overlapping and gradual transition to global chaos according to the Chirikov criterion [38].

Figures 1 and 2 demonstrate phase portraits constructed by means of map (10) with $\tau=4 \pi$ and $\tau=20 \pi$, respectively. Notably, figures corresponding to the same value of $\tau$ but different realizations of harmonic noise represent very similar patterns with nearly the same fraction of regular phase space area. In the case of $\tau=4 \pi$ (see Fig. 11), the central part of phase space maintains stability for all realizations of harmonic noise. This domain corresponds to the vicinity of the stable equilibrium point of the unperturbed system. Chaos emerges in the neighbourhood of the unperturbed separatrix. Increasing of $\tau$ to $20 \pi$ results in remarkable shrinking of the regular phase space area, as it is illustrated in Fig. 2. Phase space region corresponding to finite motion becomes submerged into the chaotic sea. Stable domains inside the unperturbed separatrix may survive only as small islands. For some realizations, they disappear completely. For example, the internal part of the chaotic sea presented in Fig. 2(d) is almost uniform, without any apparent islands. Further increasing of $\tau$ results in complete disappearance of stable islands in the phase space region enclosed by the unperturbed separatrix.

\section{Finite-time evolution operator}

Phase space portraits presented in the preceding section indicate fast destruction of stable domains. Let's consider how this process is revealed in quantum dynamics. Quantum counterpart of one-step Poincaré map is the operator $\hat{G}$ defined as

$$
\hat{G}(\tau) \bar{\Psi}(x)=\exp \left(-\frac{i}{\hbar} \hat{H} \tau\right) \bar{\Psi}(x)=\left.\Psi(x, t)\right|_{t=\tau},
$$

where $\bar{\Psi}(x)=\Psi(x, t=0)$. Hereafter we shall refer to $\hat{G}$ as the finite-time evolution operator (FTEO). Each realization of harmonic noise creates its own realization of the FTEO. FTEO was firstly utilized in [39] for the problem of noise-driven quantum diffusion. Wave analogue of the FTEO was used in [22, 23, 40,42].

Peculiarities of classical phase space should be reflected in spectral properties of the FTEO. Eigenvalues and eigenfunctions of the FTEO satisfy the equation

$$
\hat{G} \Psi_{m}(x)=g_{m} \Psi_{m}(x) \equiv e^{-i \epsilon_{m} / \hbar} \Psi_{m}(x) .
$$

Quantity $\epsilon_{m}$ is the analogue of quasienergy in time-periodic quantum systems. As increasing of $\tau$ results in destruction of regular domains, one should expect transition in statistics of level spacings $s=\epsilon_{m+1}-\epsilon_{m}$ from Poissonian to Wigner-like regime [39]. 
This expectation can fail in the presence of periodic-orbit bifurcations [22,40]. Moreover, analysis of level spacing statistics doesn't provide accurate estimate of the regular phase space area [23]. In this way, analysis of FTEO eigenfunctions seems to be more robust way. To facilitate the analysis, eigenfunctions $\Phi_{m}$ can be expanded over eigenstates of the unperturbed potential

$$
\Phi_{m}(x)=\sum_{n} c_{m n} \phi_{n}(x) .
$$

Chaos implies extensive transitions between energy levels [43], therefore, a chaos-assisted eigenfunction of FTEO should be compound of many unperturbed eigenstates. Thus, one can use the participation ratio

$$
\nu=\left(\sum_{m}\left|c_{m n}\right|^{4}\right)^{-1},
$$

as measure of "chaoticity". Phase space region associated with an eigenfunction can be found by means of the parameter [44]

$$
\mu=\sum_{m=1}^{M}\left|c_{m n}\right|^{2} m .
$$

Indeed, the formula $\langle I\rangle=\hbar(\mu+1 / 2)$ yields the expectation value of the classical action corresponding to the eigenfunction. Parameters $\nu$ and $\mu$ provide suitable classification of eigenfunctions and can be used for tracking the transition from order to chaos by means of numerical simulation.
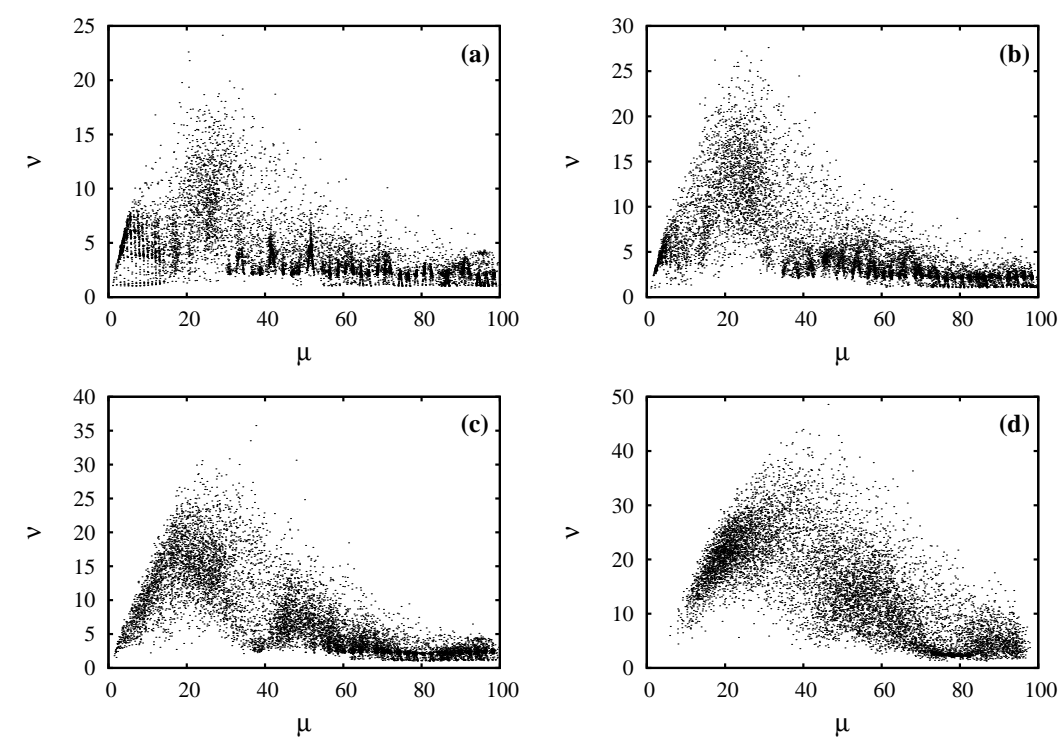

Figure 3. Distribution of FTEO eigenfunctions in the $\mu-\nu$ plane. Values of $\tau$ : (a) $4 \pi$, (b) $10 \pi$, (c) $20 \pi$, (d) $100 \pi$.

Numerical simulation was conducted with the ensemble of 100 realizations of the FTEO. 100 eigenfunctions with the lowest values of $\mu$ are taken into account for each 
realization. An informative view is provided by distributions of FTEO eigenfunctions in the $\mu-\nu$ space. These distributions corresponding to different values of $\tau$ are presented in Fig. 3. For relatively small values of $\tau$, the dots corresponding to eigenfunctions form ordered patterns consisted of distinct slightly biased lines. Such patterns were earlier observed in [23], where they were called "stalagmites". Each "stalagmite" is formed by eigenfunctions localized near periodic orbits of the one-step Poincaré with the same location in the action space. Transition to chaos is accompanied by delocalization of eigenfunctions and smearing of "stalagmites". It should be noted that smearing is partially suppressed by dynamical localization [18], that is, weakly unstable periodic orbits retain the ability to trap eigenfunctions [45]. For $\tau=4 \pi$, stalagmites occur in the whole range of $\mu$ values, except for the vicinity of $\mu=25$. This value of $\mu$ corresponds to the phase space region near the unperturbed separatrix of the pendulum, where the classical chaotic sea is originated initially. As $\tau$ increases, the smeared domain grows indicating gradual transition to chaos due to overlapping of classical resonances (13). Notably, chaos-assisted delocalization firstly emerges in the range of small values of $\mu$, corresponding to finite motion. This infers efficient destruction of invariant curves impeding transition between finite and infinite regimes. Results of Ref. [27] show that the destruction of invariant curves leads to the onset of directed current.

Nevertheless, traces of eigenfunctions with good persistence to chaos are visible even for $\tau=100 \pi$. For instance, there is a small slightly smeared horizontal stripe near $\mu \simeq 80$ (see Fig. 3 (d)) corresponding to infinite motion with relatively high velocities. That phase space region is characterized by inequality $T \ll \tau$ that anticipates weak influence of resonances (13), and, hence, weakness of chaos induced by their overlapping.

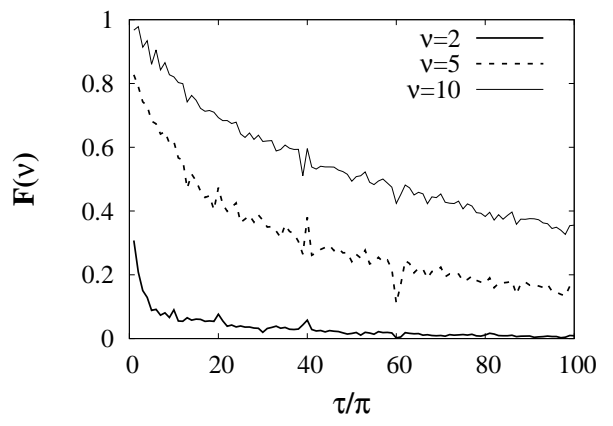

Figure 4. Fractions of eigenfunction ensemble, corresponding to different regimes of localization, vs time.

As domains of finite-time stability in phase space give rise to FTEO eigenfunctions with small $\nu$, one can estimate their contribution using the cumulative distribution

$$
F(\nu)=\int_{1}^{\nu} \rho\left(\nu^{\prime}\right) d \nu^{\prime},
$$

where $\rho\left(\nu^{\prime}\right)$ is the corresponding probability density function. We refer to the case $\nu \leq 2$ as the regime of strong localization. Indeed, inequality $\nu \leq 2$ implies that a 
FTEO eigenfunction is mainly contributed from one or two unperturbed eigenstates. In addition, let's consider two regimes of moderate localization: $\nu \leq 5$ and $\nu \leq 10$. The sense of the latter two criteria becomes apparent if we take into account that finite motion corresponds to the 25 lowest unperturbed eigenstates. Thus, the inequality $\nu \leq 5$ $(\nu \leq 10)$ picks out FTEO eigenfunctions occupying less than 20 percents (40 percents) of the phase space area enclosed by the unperturbed separatrix. As it follows from Fig. 4, fraction of strongly localized eigenfunctions rapidly decays down to nearly zero. It indicates the absence of significant long-living regular domains in classical phase space. It should be mentioned that the opposite situation was observed in Ref. [23], where slow decay of $F(2)$ was linked to the presence of degenerate tori in phase space. In the present case there is no degenerate tori, therefore, there is no such route to the persistence of regular domains. Fractions of moderately localized functions decay much slower, and their impact remains significant even at $\tau=100 \pi$. It can be understood as manifestation of dynamical localization, i. e. partial suppression of chaos-assisted diffusion due to the interference.

\section{Conclusion}

In the present paper, we demonstrate approach designed for studying quantum manifestations of classical finite-time stability under weak external random perturbation. The approach is based on construction of the finite-time evolution operator (FTEO). It is emphasized that statistical properties of FTEO eigenfunctions are closely linked to classical phase space structure revealed by one-step Poincaré map that can be regarded as the classical counterpart of the FTEO. In particular, the stalagmite-like patterns on distributions of eigenfunctions in the space of parameters $\mu$ and $\nu$ are related to wavefunction concentrations near the periodic orbits of the one-step Poincaré map. Increasing of time results in emergence of chaos, therefore, periodic orbits loss their stability. This leads to smearing of the "stalagmites". Chaos-assisted destruction of "stalagmites" represents an alternative view onto the order-to-chaos transition in randomly-driven quantum systems.

In the present work, we consider stable domains satisfying the condition of invariance under translation over finite time interval. They occur owing to the same mechanism as stable islands in time-periodic systems. Namely, they are remnants of nonlinear resonances of one-step Poincare map and arise in somewhat regular and predictable way. To underline the latter circumstance, we present phase space portraits corresponding to different realizations, and one can see that regular domains appear in the same ranges of the action (or energy) values.

This work is supported by the Russian Foundation of Basic Research under project 13-01-12404, and by the Siberian and Far-Eastern Branches of the Russian Academy of Sciences under joint project 12-II-SO-07-022. Authors are grateful to Michael Uleysky for the assistance in preparation of the manuscript. 
Order-to-chaos transition

\section{References}

[1] Tomsovic S and Brown M G 2010 Ocean acoustics: a novel laboratory for wave chaos (Cambridge University Press) pp 169-187

[2] Abdullaev S S 2011 Phys. Rev. E 84026204

[3] Wolfson M A and Tomsovic S 2001 J. Acoust. Soc. Am. 109 2693-2703

[4] Laffargue T, Lam K D N T, Kurchan J and Tailleur J 2013 J. Phys. A: Math. Theor. 46254002

[5] Topinka M, LeRoy B, Shaw S, Heller E, Westervelt R, Maranowski K and Gossard A 2000 Science $2892323-2326$

[6] Topinka M, LeRoy B, Westervelt R, Shaw S, Fleischmann R, Heller E, Maranowski K and Gossard A 2001 Nature 410 183-186

[7] Kaplan L 2002 Phys. Rev. Lett. 89184103

[8] Liu B and Heller E J 2013 Phys. Rev. Lett. 111236804

[9] Makarov D V, Uleysky M Y and Prants S V 2004 Chaos 14 79-95

[10] Klyatskin V I and Gurarie D 1999 Physics-Uspekhi 42 165-198

[11] Prants S, Budyansky M, Ponomarev V and Uleysky M 2011 Ocean Modelling $38114-125$

[12] Finn J and Apte S V 2013 Chaos 23013145

[13] Froyland G, Padberg K, England M H and Treguier A M 2007 Phys. Rev. Lett. 98224503

[14] Zaslavsky G M 2007 The physics of chaos in Hamiltonian systems (London: Imperial College Press)

[15] Zaslavsky G M 2002 Physica D: Nonlinear Phenomena 168 292-304

[16] Tomsovic S and Lakshminarayan A 2007 Phys. Rev. E 76036207

[17] Gutzwiller M C 1990 Chaos in classical and quantum mechanics (Springer)

[18] Stöckmann H J 2007 Quantum Chaos: An Introduction (Cambridge: Cambridge University Press)

[19] Heller E J 1984 Phys. Rev. Lett. 53 1515-1518

[20] Makarov D and Uleysky M 2006 J. Phys. A: Math. Gen. 39 489-497

[21] Makarov D V, Uleysky M Y, Budyansky M V and Prants S V 2006 Phys. Rev. E 73066210

[22] Virovlyansky A L, Makarov D V and Prants S V 2012 Physics-Uspekhi 55 18-46

[23] Makarov D V, Kon'kov L E, Uleysky M Y and Petrov P S 2013 Phys. Rev. E 87012911

[24] Singh K P, Kenfack A and Rost J M 2008 Phys. Rev. A 77022707

[25] Kenfack A, Rost J M and Großmann F 2008 New J. Phys. 10013020

[26] Feng L Q and Chu T S 2012 J. Mol. Model. 18 5097-5106

[27] Makarov D V and Kon'kov L E 2013 Physics Letters A 377 3093-3097

[28] Neiman A and Schimansky-Geier L 1994 Phys. Rev. Lett. 72 2988-2991

[29] Anishchenko V S, Neiman A B, Moss F and Schimansky-Geier L 1999 Physics-Uspekhi 42 7-36

[30] Makarov D and Uleysky M 2006 JETP Lett. 83 522-525

[31] Makarov D V and Uleysky M Y 2007 Phys. Rev. E 75065201

[32] Makarov D V 2008 Tech. Phys. Lett. 34 303-305

[33] Makarov D V, Sosedko E V and Uleysky M Y 2010 Europ. Phys. J. B 73 571-579

[34] Greenaway M T, Balanov A G and Fromhold T M 2013 Phys. Rev. A 87013411

[35] Gan C, Wang Q and Perc M 2010 J. Phys. A: Math. Theor. 43125102

[36] Hitczenko P and Medvedev G S 2013 J. Nonl. Sci. 23 835-861

[37] Berglund N, Gentz B and Kuehn C 2013 arXiv preprint arXiv:1312.6353

[38] Chirikov B V 1979 Phys. Rep. 52263 - 379

[39] Kolovsky A R 1997 Phys. Rev. E 56 2261-2264

[40] Makarov D V, Kon'kov L E and Uleysky M Y 2010 arXiv preprint arXiv:1008.3037

[41] Hegewisch K C and Tomsovic S 2012 Europhys. Lett. 9734002

[42] Hegewisch K C and Tomsovic S 2013 J. Acoust. Soc. Am. 134 3174-3184

[43] Berman G P and Kolovskii A R 1992 Sov. Phys. Usp. 35 303-326

[44] Smirnov I P, Virovlyansky A L, Edelman M and Zaslavsky G M 2005 Phys. Rev. E 72026206

[45] Kon'kov L E, Makarov D V, Sosedko E V and Uleysky M Y 2007 Phys. Rev. E 76056212 